\title{
Association of genetic polymorphisms in vascular endothelial growth factor with susceptibility to coronary artery disease: a meta-analysis
}

\author{
Wen-Qi Ma@, Ying Wang, Xi-Qiong Han, Yi Zhu and Nai-Feng Liu*
}

\begin{abstract}
Background: Single nucleotide polymorphisms (SNPs) located in the vascular endothelial growth factor (VEGF) gene may be correlated with the susceptibility to coronary artery disease (CAD) - although results have been controversial. The aim of this meta-analysis is to clarify the effects of VEGF -2578A/C (rs699947), -1154G/A (rs1570360), +405C/G (rs2010963), and + 936C/T (rs3025039) polymorphisms on CAD risk.
\end{abstract}

Methods: Pooled odds ratio (OR) and corresponding 95\% confidence intervals (Cls) were calculated to estimate the strength of the association between VEGF gene polymorphisms and CAD risk. Fixed- or random-effects model was used depending on the heterogeneity between studies.

Results: In total, 13 eligible articles containing 29 studies were analysed. The pooled analysis indicated that the VEGF gene polymorphisms of rs699947, rs2010963, and rs3025039 were associated with an increased risk of CAD, whereas no significant associations were observed with the rs1570360 polymorphism. A subgroup analysis stratified by ethnicity revealed that the rs699947 and rs3025039 polymorphisms were associated with CAD risk in Asian populations. In addition, stratification by control source indicated an increased risk of CAD susceptibility with the rs699947 polymorphism for population-based studies of reduced heterogeneity.

Conclusions: In summary, we concluded that the VEGF gene polymorphisms rs699947, rs2010963, and rs3025039 are correlated with an elevated CAD risk.

Keywords: Vascular endothelial growth factor, Coronary artery disease, Gene polymorphisms, Meta-analysis

\section{Background}

Coronary artery disease $(\mathrm{CAD})$ is and will remain the main cause of morbidity and mortality worldwide [1]. CAD is a chronic, progressive, and polygenic disease, and atherosclerosis is appears to be the major pathophysiological process underlying $\mathrm{CAD}$ [2]. The roles of endothelial dysfunction and angiogenesis in atherosclerosis development have been widely reported [3, 4]. Endothelial dysfunction, which is frequently triggered by smoking, dyslipidaemia, hypertension, hyperglycaemia, and insulin resistance, may influence the balance between endothelium-dependent vasoconstriction and vasodilatation, the upregulation of cytokines, adhesion

\footnotetext{
* Correspondence: liunf@seu.edu.cn

Department of Cardiology, Zhongda Hospital, School of Medicine, Southeast University, 87 Dingjiaqiao, Nanjing 210009, People's Republic of China
}

molecule expression, leukocyte and monocyte migration, and platelet activation [5, 6]. Moreover, angiogenesis may be related to disturbances in endothelial cell physiology. These complex, associated processes of endothelial dysfunction and angiogenesis require the participation of various growth factors.

Vascular endothelial growth factor (VEGF), an essential component of angiogenesis, has been reported to induce endothelial cell migration and proliferation, enhance vascular permeability, and modulate thrombogenicity [7-9]. The VEGF family includes VEGF-A, VEGF-B, VEGF-C, VEGF-D, VEGF-E, VEGF-F, and placental growth factor, and all these growth factors perform their functions by interacting with high-affinity receptor tyrosine kinases [10]. The VEGF gene is located in chromosome 6p21.3

(c) The Author(s). 2018 Open Access This article is distributed under the terms of the Creative Commons Attribution 4.0 International License (http://creativecommons.org/licenses/by/4.0/), which permits unrestricted use, distribution, and reproduction in any medium, provided you give appropriate credit to the original author(s) and the source, provide a link to the Creative Commons license, and indicate if changes were made. The Creative Commons Public Domain Dedication waiver (http://creativecommons.org/publicdomain/zero/1.0/) applies to the data made available in this article, unless otherwise stated. 
and contains a 14-kb coding region with eight exons and seven introns [11]. It is expressed in various cell types, such as endothelial cells, vascular smooth muscle cells, macrophages, and several tumour cells. Molecular biology studies have confirmed that VEGF expression is regulated by certain single nucleotide polymorphisms (SNPs), which have tissue- and age-specific expression patterns [12-15]. Furthermore, VEGF gene variability may be of particular interest for many angiogenesis-associated diseases, such as tumours, osteosarcoma, age-related macular degeneration, diabetic retinopathy, and chronic immune-mediated inflammatory diseases [16-20].

Some of these polymorphisms, including VEGF -2578A/ C (rs699947), -1154G/A (rs1570360), +405C/G (rs2010963), and $+936 \mathrm{C} / \mathrm{T}$ (rs3025039), which arise from the vascular expression of different VEGF proteins, have been associated with CAD susceptibility; however, these findings are controversial. For example, Han et al. [21] indicated that two VEGF SNPs (rs2010963 and rs3025039) were associated with CAD susceptibility in a Chinese population. Similarly, $\mathrm{Li}$ et al. [22] also reported that the $\mathrm{C}$ allele of VEGF (rs699947) may be an important independent risk factor for susceptibility to CAD. However, other studies have drawn opposite conclusions. Biselli et al. [23] suggested a possible protective effect of the rs699947 polymorphism on CAD severity because of a reduced VEGF expression.

The existence of a correlation between VEGF gene SNPs and CAD susceptibility remains controversial and inconclusive. Moreover, no relevant meta-analyses have been published. Consequently, we performed a metaanalysis to investigate the association between VEGF gene polymorphisms and CAD risk.

\section{Methods}

\section{Literature search strategy}

The literature search was conducted by two authors (Ma and Han). PubMed, EMBASE, Web of Science, ScienceDirect, and Cochrane Library were systematically searched. The keywords and terms used for the searches included the following: "vascular endothelial growth factor" OR "VEGF" OR “-2578 A/C” OR “+405C/G” OR “+936C/T” OR “1154G/A" OR "rs699947" OR "rs3025039" OR "rs2010963" OR "rs1570360"; "genetic polymorphism" OR "mutation" OR "variant" OR "genotype"; and "angina" OR "myocardial infarction" OR "atherosclerosis" OR "acute coronary syndrome" OR "coronary artery disease" OR "coronary heart disease". Hand-searching of reference lists in relevant articles was also performed. The last search was conducted on October 24, 2017.

\section{Inclusion and exclusion criteria}

Eligible studies in our meta-analysis complied with the following criteria: 1) case-control studies evaluating VEGF gene polymorphisms (rs699947, rs2010963, rs3025039, and rs1570360) and CAD susceptibility; 2) all CAD cases were documented by angiographic evidence showing at least $50 \%$ stenosis of one major coronary artery, myocardial infarction (MI), or coronary artery bypass surgery; 3) sufficient published data, such as the total number of cases and controls, distribution of genotypes, and other relevant information; and 4) language was restricted to English. Studies were excluded if they met the following criteria: 1) letters to the editor, abstracts, animal studies, or reviews; 2) data overlapping with previous publications; and 3) studies with unusable or insufficient data.

\section{Data extraction}

Following the Meta-analysis of Observational Studies in Epidemiology (MOOSE) guidelines for reporting metaanalyses of observational studies (Additional file 1: Table S1), data from eligible studies were separately extracted by two authors (Ma and $\mathrm{Zhu}$ ), and eligibility disagreements were discussed and resolved by a third author (Liu). For each eligible study, data included information regarding the author, year of publication, number of cases and controls, country, ethnicity, genotyping methods, genotype frequency in cases and controls, sources of controls, and Hardy-Weinberg equilibrium (HWE) in controls.

\section{Quality assessment}

The Newcastle-Ottawa Scale (NOS) quality score was applied to assess the quality of each eligible study. Validated quality assessment consisted of three parameters, including selection, comparability, and exposure. NOS scores ranged from 0 and 9 stars. Studies with an NOS score of five or greater were deemed moderate to high quality, whereas studies with an NOS score of less than five were considered low quality.

\section{Statistics analysis}

All calculations and graphs were performed by Review Manager v5.2 (The Cochrane Collaboration, Oxford, UK) and Stata 12.0 (Stata Corporation, College Station, Texas, USA). Pooled odds ratios (ORs) and corresponding 95\% confidence intervals (CIs) were selected to estimate the strength of the association. For the VEGF rs6699947 polymorphism, pooled ORs were obtained for dominant $(\mathrm{CC}+$ $A C$ vs. $A A)$, recessive (CC vs. $A A+A C)$, homozygous (CC vs. AA), heterozygous (AC vs. AA), and allele (C vs. A) genetic models. Similar genetic models were also used to assess the rs2010963, rs3025039, and rs1570360 polymorphisms. The Cochrane $\mathrm{Q}$-test and index $\left(\mathrm{I}^{2}\right)$ were used to assess the heterogeneity within studies. A Q-test with $P<0.10$ indicated significant heterogeneity. $\mathrm{I}^{2}$ values of $0-25,25-$ $50 \%$, and $>50 \%$ represented mild, moderate, and high-level heterogeneity, respectively. A fixed- or random-effects model was used to calculate OR and 95\% CIs based on the study heterogeneity strength. Subsequently, a subgroup 
analysis was performed to search for potential sources of heterogeneity. If there was an appropriate number of included studies, subgroup analyses based on ethnicity (Asian and Caucasian populations), control source (populationbased and hospital-based controls), and sample size (studies with more than 500 subjects were categorized as "large" and studies with less 500 subjects were categorized as "small"), and the type of CAD were performed to detect the sources of the heterogeneity. A sensitivity analysis was performed to assess the stability of the individual studies. Possible publication bias was assessed using funnel plots and Egger's linear regression test.

\section{Results}

\section{Study selection and characteristics}

A total of 1488 articles were retrieved following the initial search. Of these publications, 915 were excluded due to duplicate records, 33 articles were determined to be ineligible after the screening of the titles and abstracts, and 20 articles were excluded after reading the full texts because of insufficient data, ineligible samples, or by virtue of being a review or conference abstract. Ultimately, thirteen eligible articles containing 29 studies met our inclusion criteria [21-33]. The study selection process is summarized in Fig. 1. The quality of the included studies was evaluated using the NOS quality score (Additional file 1: Table S2).

The data from the eligible studies are displayed in Table 1. Eight studies containing 2471 cases and 2811 controls found an association between the VEGF rs699947 polymorphism and susceptibility to CAD. Ten studies involving 2303 cases and 2862 controls focused on the relationship between the VEGF rs2010963 polymorphism and susceptibility to CAD. Eight studies of the VEGF rs3025039 polymorphism included 2136 cases and 2477 controls, and three VEGF rs1570360 polymorphism studies included 1227 cases and 1166 controls. The group of controls consisted of healthy volunteers from the community or patients who underwent health examinations in the hospital. Twelve studies were based on Caucasian samples, and seventeen studies used Asian samples. The countries in which the eligible studies were conducted included Brazil, China, Finland, Iran, Slovenia, and the United Kingdom. The distribution of genotypes in the controls was tested in 29 studies and found to be mostly consistent with HWE, except for two studies [31].

\section{Association between the rs699947 polymorphism and susceptibility to CAD}

For all studies, the meta-analysis showed an increased risk between the rs699947 polymorphism and CAD susceptibility in the heterozygous genetic model (AC vs. AA: OR $=1.26,95 \% \mathrm{CI}=1.10-1.45)$ with a low between-study heterogeneity. No significant associations were observed

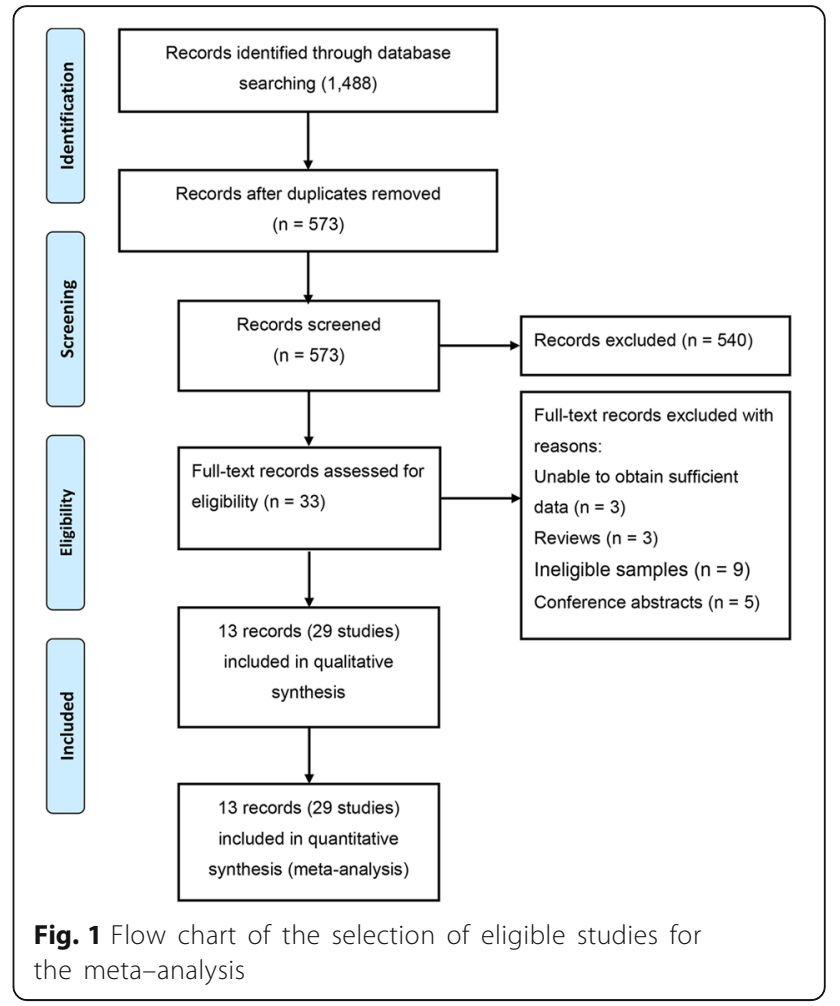

in the dominant $(\mathrm{CC}+\mathrm{AC}$ vs. $\mathrm{AA}: \mathrm{OR}=1.17,95 \% \mathrm{CI}$ : 0.93-1.47), recessive (CC vs. $A A+A C: O R=0.96,95 \%$ CI: 0.71-1.31), homozygous (CC vs. AA: $\mathrm{OR}=1.05,95 \%$ CI: $0.71-1.55$ ), and allele ( $\mathrm{C}$ vs. A: $\mathrm{OR}=1.01,95 \% \mathrm{CI}$ : 0.83-1.23) genetic models (Table 2; Additional file 1: Figs. S1-S5). Stratification by ethnicity indicated that the rs699947 polymorphism was significantly associated with CAD risk in Asian populations compared to Caucasian populations under the heterozygous genetic model (AC vs. $\mathrm{AA}: \mathrm{OR}=1.25,95 \% \mathrm{CI}=1.07-1.46$ ). In the subgroup analysis stratified by the control source, we observed a significantly increased risk of CAD susceptibility in the dominant genetic model $(\mathrm{CC}+\mathrm{AC}$ vs. $\mathrm{AA}: \mathrm{OR}=1.38,95 \% \mathrm{CI}$ : 1.19-1.61) within population-based studies with reduced heterogeneity. A similar result was also detected for the heterozygous genetic model. When we conducted a subgroup analysis by sample size, the same significant associations were observed in studies with large sample sizes in dominant and heterozygous genetic models with a low heterogeneity (Table 2).

\section{Association between the rs2010963 polymorphism and susceptibility to CAD}

A significant association between rs2010963 polymorphism and CAD risk was found under the recessive and homozygous genetic models (CC vs. GG + GC: OR $=1.45,95 \% \mathrm{CI}=1.03-2.05 ; \mathrm{CC}$ vs. $\mathrm{GG}: \mathrm{OR}=1.57,95 \%$ $\mathrm{CI}=1.02-2.42) \quad$ (Table 3; Additional file 1: Figs. S6-S10). Stratification by sample size indicated that the 
Table 1 Characteristics of the individual studies included in the meta-analysis

\begin{tabular}{|c|c|c|c|c|c|c|c|c|c|c|}
\hline \multirow[t]{2}{*}{ Study } & \multirow[t]{2}{*}{ Country } & \multirow[t]{2}{*}{ Ethnicity } & \multirow[t]{2}{*}{ Disease } & \multirow{2}{*}{$\begin{array}{l}\text { Control } \\
\text { Source }\end{array}$} & \multirow{2}{*}{$\begin{array}{l}\text { Genotyping } \\
\text { Methods }\end{array}$} & \multirow{2}{*}{$\begin{array}{l}\text { Sample } \\
\text { Size } \\
\text { Cases/ } \\
\text { Controls }\end{array}$} & \multicolumn{2}{|c|}{ Genotype Distribution } & \multirow[t]{2}{*}{$P_{\text {HWE }}$} & \multirow[t]{2}{*}{ NOS } \\
\hline & & & & & & & Cases & Controls & & \\
\hline rs699947 & & & & & & & $\mathrm{AA} / \mathrm{AC} / \mathrm{CC}$ & $\mathrm{AA} / \mathrm{AC} / \mathrm{CC}$ & & \\
\hline Biselli $2008^{23}$ & Brazil & Caucasian & CAD & $\mathrm{HB}$ & PCR & $175 / 108$ & $32 / 96 / 47$ & $27 / 51 / 30$ & 0.569 & 6 \\
\hline Kangas-Kontio $2009^{25}$ & Finland & Caucasian & $\mathrm{Ml}$ & $\mathrm{HB}$ & TaqMan & $188 / 218$ & $64 / 87 / 37$ & 77/101/40 & 0.498 & 7 \\
\hline Chen $2011^{26}$ & UK & Caucasian & Ml & PB & PCR-RFLP & $46 / 372$ & $10 / 34 / 2$ & 100/167/105 & 0.050 & 8 \\
\hline Amoli $2012^{27}$ & Iran & Asian & CAD & $\mathrm{HB}$ & PCR & $50 / 50$ & $9 / 26 / 15$ & $14 / 27 / 9$ & 0.520 & 6 \\
\hline Cui $2013^{28}$ & China & Asian & CAD & $\mathrm{HB}$ & PCR & $242 / 253$ & 27/78/137 & $12 / 69 / 172$ & 0.148 & 6 \\
\hline Gu $2013^{29}$ & China & Asian & CAD & $\mathrm{HB}$ & MassARRAY & $427 / 472$ & $30 / 178 / 219$ & $31 / 174 / 267$ & 0.713 & 6 \\
\hline Li $2016^{22}$ & China & Asian & $C A D$ & PB & PCR & $533 / 533$ & $180 / 250 / 103$ & $217 / 237 / 79$ & 0.280 & 8 \\
\hline Liu $2016^{31}$ & China & Asian & CAD & PB & PCR & $810 / 805$ & $275 / 381 / 154$ & $339 / 345 / 121$ & 0.03 & 8 \\
\hline rs2010963 & & & & & & & GG/GC/CC & GG/GC/CC & & \\
\hline Petrovic $2007^{24}$ & Slovenia & Caucasian & $\mathrm{Ml}$ & PB & PCR & $143 / 228$ & $42 / 76 / 25$ & $103 / 104 / 21$ & 0.470 & 7 \\
\hline Kangas-Kontio $2009^{25}$ & Finland & Caucasian & $\mathrm{Ml}$ & $H B$ & TaqMan & $186 / 218$ & $113 / 61 / 12$ & $143 / 67 / 8$ & 0.966 & 7 \\
\hline Chen $2011^{26}$ & UK & Caucasian & $\mathrm{Ml}$ & PB & PCR-RFLP & $46 / 372$ & 26/19/1 & $174 / 159 / 39$ & 0.765 & 8 \\
\hline Cui $2013^{28}$ & China & Asian & $C A D$ & $\mathrm{HB}$ & PCR & $242 / 253$ & $75 / 102 / 65$ & $104 / 114 / 35$ & 0.675 & 6 \\
\hline Gu $2013^{29}$ & China & Asian & CAD & $\mathrm{HB}$ & MassARRAY & $419 / 468$ & $144 / 215 / 60$ & $154 / 225 / 89$ & 0.672 & 6 \\
\hline Douvaras $2013^{32}$ & Greece & Caucasian & Ml & $H B$ & PCR-RFLP & $102 / 98$ & $37 / 49 / 16$ & $29 / 55 / 14$ & $>0.050$ & 7 \\
\hline Moradzadegan $2015^{30}$ & Iran & Asian & CAD & $\mathrm{HB}$ & PCR-RFLP & $141 / 369$ & $43 / 65 / 33$ & $85 / 197 / 87$ & 0.193 & 7 \\
\hline Han $2015^{21}$ & China & Asian & $C A D$ & $\mathrm{HB}$ & MassARRAY & $144 / 150$ & $69 / 49 / 26$ & $86 / 54 / 10$ & 0.701 & 6 \\
\hline Li $2016^{22}$ & China & Asian & $C A D$ & PB & PCR & $533 / 533$ & $50 / 233 / 250$ & $71 / 239 / 223$ & 0.583 & 8 \\
\hline Nia $2017^{33}$ & Iran & Asian & $C A D$ & $\mathrm{HB}$ & TaqMan & $347 / 173$ & $167 / 135 / 45$ & $102 / 63 / 8$ & $>0.050$ & 7 \\
\hline rs3025039 & & & & & & & $\mathrm{CC} / \mathrm{CT} / \mathrm{TT}$ & $\mathrm{CC} / \mathrm{CT} / \mathrm{TT}$ & & \\
\hline Biselli $2008^{23}$ & Brazil & Caucasian & CAD & $\mathrm{HB}$ & PCR & 175/108 & $133 / 36 / 6$ & $83 / 23 / 2$ & 0.783 & 6 \\
\hline Kangas-Kontio $2009^{25}$ & Finland & Caucasian & $\mathrm{Ml}$ & $H B$ & TaqMan & $187 / 218$ & $140 / 42 / 5$ & $155 / 56 / 7$ & 0.488 & 7 \\
\hline Chen $2011^{26}$ & UK & Caucasian & $\mathrm{Ml}$ & PB & PCR-RFLP & $46 / 372$ & $37 / 8 / 1$ & $264 / 95 / 13$ & 0.229 & 8 \\
\hline Cui $2013^{28}$ & China & Asian & $C A D$ & $H B$ & PCR & $242 / 253$ & $133 / 95 / 14$ & $159 / 86 / 8$ & 0.373 & 6 \\
\hline Gu $2013^{29}$ & China & Asian & CAD & $\mathrm{HB}$ & MassARRAY & $430 / 473$ & $272 / 142 / 16$ & $300 / 159 / 14$ & 0.194 & 6 \\
\hline Douvaras $2013^{32}$ & Greece & Caucasian & $\mathrm{Ml}$ & $H B$ & PCR-RFLP & $102 / 98$ & $68 / 30 / 4$ & $69 / 27 / 2$ & $>0.050$ & 7 \\
\hline $\operatorname{Han} 2015^{21}$ & China & Asian & CAD & $\mathrm{HB}$ & MassARRAY & $144 / 150$ & $84 / 55 / 5$ & $115 / 31 / 4$ & 0.290 & 6 \\
\hline Liu $2016^{31}$ & China & Asian & $C A D$ & PB & PCR & $810 / 805$ & $472 / 308 / 30$ & $617 / 167 / 21$ & 0.020 & 8 \\
\hline rs1570360 & & & & & & & GG/GA/AA & GG/GA/AA & & \\
\hline Biselli $2008^{23}$ & Brazil & Caucasian & $C A D$ & $\mathrm{HB}$ & $P C R$ & 175/108 & $96 / 61 / 18$ & $57 / 38 / 13$ & 0.104 & 6 \\
\hline Cui $2013^{28}$ & China & Asian & $C A D$ & $\mathrm{HB}$ & $P C R$ & $242 / 253$ & $151 / 79 / 12$ & $172 / 69 / 12$ & 0.148 & 6 \\
\hline Liu $2016^{31}$ & China & Asian & CAD & PB & $P C R$ & $810 / 805$ & $54 / 370 / 386$ & $137 / 456 / 212$ & $>0.050$ & 8 \\
\hline
\end{tabular}

Abbreviations: $C A D$ coronary artery disease; $M I$ myocardial infarction; $P C R$ polymerase chain reaction; $P C R-R F L P$ polymerase chain reaction-restriction fragment length polymorphism; HWE Hardy-Weinberg equilibrium; $P B$ population-based; HB hospital-based; NOS Newcastle-Ottawa quality scale; UK the United Kingdom

rs2010963 polymorphism was significantly associated with CAD risk for small sample sizes compared to large sample sizes in several genetic models (CC vs. GG + $\mathrm{GC}: \mathrm{OR}=1.52,95 \% \mathrm{CI}=1.01-2.33$; $\mathrm{CC}$ vs. $\mathrm{GG}: \mathrm{OR}=$ 2.03, 95\% CI $=1.26-3.28$; $\mathrm{C}$ vs. $\mathrm{G}$ : $\mathrm{OR}=1.27,95 \% \mathrm{CI}=$ 1.03-1.78). No significant associations were observed following a subgroup analysis by ethnicity and control source (Table 3).

\section{Association between the rs3025039 polymorphism and susceptibility to CAD}

Meta-analysis of the rs3025039 polymorphism showed an elevated risk of CAD in the homozygous genetic model (TT vs. $\mathrm{CC}$ : $\mathrm{OR}=1.55,95 \% \mathrm{CI}=1.10-2.17$ ) with a low between-study heterogeneity. No significant associations were observed in the remaining genetic models (TT+ CT vs. $\mathrm{CC}$ : $\mathrm{OR}=1.26,95 \% \mathrm{CI}: 0.89-1.79$; TT vs. $\mathrm{CC}+\mathrm{CT}$ : OR 
Table 2 Summary of odds ratios (95\% Cl) in the analysis of the relationship between VEGF gene polymorphisms in rs699947 and coronary artery disease susceptibility

\begin{tabular}{|c|c|c|c|c|c|c|c|}
\hline \multirow[t]{2}{*}{ Genetic Model } & \multirow{2}{*}{$\begin{array}{l}\text { Overall and } \\
\text { Subgroups }\end{array}$} & \multirow[t]{2}{*}{$\mathrm{N}$} & \multicolumn{3}{|c|}{ Test of Association } & \multicolumn{2}{|c|}{ Test of Heterogeneity } \\
\hline & & & $\overline{\mathrm{OR}}$ & $95 \% \mathrm{Cl}$ & $P_{\text {-value }}$ & $P_{\text {Heterogeneity }}$ & $P^{2}(\%)$ \\
\hline & Overall & 8 & 1.17 & $0.93,1.47$ & 0.170 & 0.040 & $52 \%$ \\
\hline & HWE (yes) & 7 & 1.10 & $0.83,1.47$ & 0.520 & 0.050 & $53 \%$ \\
\hline & PB & 3 & 1.38 & $1.19,1.61$ & 0.000 & 0.950 & $0 \%$ \\
\hline \multirow[t]{8}{*}{$C C+A C$ vs. AA } & $\mathrm{HB}$ & 5 & 0.99 & $0.65,1.51$ & 0.970 & 0.040 & $65 \%$ \\
\hline & Large sample size & 3 & 1.34 & $1.16,1.56$ & 0.000 & 0.340 & $8 \%$ \\
\hline & Small sample size & 5 & 1.06 & $0.67,1.68$ & 0.800 & 0.040 & $61 \%$ \\
\hline & Asians & 5 & 1.11 & $0.79,1.55$ & 0.550 & 0.009 & $71 \%$ \\
\hline & Caucasians & 3 & 1.21 & $0.89,1.64$ & 0.220 & 0.620 & $0 \%$ \\
\hline & Overall & 8 & 0.96 & $0.71,1.31$ & 0.800 & 0.000 & $75 \%$ \\
\hline & HWE (yes) & 7 & 0.90 & $0.63,1.27$ & 0.550 & 0.000 & $74 \%$ \\
\hline & PB & 3 & 0.98 & $0.53,1.80$ & 0.950 & 0.003 & $83 \%$ \\
\hline \multirow[t]{8}{*}{$C C$ vs. $A A+A C$} & $\mathrm{HB}$ & 5 & 0.87 & $0.66,1.14$ & 0.320 & 0.130 & 45 \\
\hline & Large sample & 3 & 1.13 & $0.80,1.60$ & 0.480 & 0.010 & $78 \%$ \\
\hline & Small sample & 5 & 0.80 & $0.47,1.37$ & 0.420 & 0.006 & $73 \%$ \\
\hline & Asians & 5 & 0.99 & $0.74,1.48$ & 0.790 & 0.000 & $79 \%$ \\
\hline & Caucasians & 3 & 0.65 & $0.27,1.56$ & 0.340 & 0.010 & $78 \%$ \\
\hline & Overall & 8 & 1.26 & $1.10,1.45$ & 0.001 & 0.210 & $27 \%$ \\
\hline & HWE (yes) & 7 & 1.20 & $1.00,1.44$ & 0.050 & 0.180 & $32 \%$ \\
\hline & PB & 3 & 1.36 & $1.15,1.60$ & 0.000 & 0.510 & $0 \%$ \\
\hline \multirow[t]{8}{*}{$A C$ vs. $A A$} & $\mathrm{HB}$ & 5 & 1.05 & $0.81,1.37$ & 0.710 & 0.210 & $32 \%$ \\
\hline & Large sample & 3 & 1.30 & $1.11,1.52$ & 0.001 & 0.680 & $0 \%$ \\
\hline & Small sample & 5 & 1.16 & $0.88,1.54$ & 0.290 & 0.080 & $53 \%$ \\
\hline & Asians & 5 & 1.25 & $1.07,1.46$ & 0.005 & 0.150 & $41 \%$ \\
\hline & Caucasians & 3 & 1.33 & $0.97,1.83$ & 0.080 & 0.250 & $29 \%$ \\
\hline & Overall & 8 & 1.05 & $0.71,1.55$ & 0.810 & 0.001 & $72 \%$ \\
\hline & HWE (yes) & 7 & 0.95 & $0.59,1.53$ & 0.770 & 0.001 & $72 \%$ \\
\hline & PB & 3 & 1.29 & $0.77,2.17$ & 0.340 & 0.030 & $72 \%$ \\
\hline \multirow[t]{8}{*}{ CC vs. AA } & $\mathrm{HB}$ & 5 & 0.95 & $0.57,1.60$ & 0.850 & 0.020 & $65 \%$ \\
\hline & Large sample & 3 & 1.36 & $0.99,1.88$ & 0.060 & 0.110 & $54 \%$ \\
\hline & Small sample & 5 & 0.82 & $0.39,1.71$ & 0.600 & 0.004 & $74 \%$ \\
\hline & Asians & 5 & 1.12 & $0.68,1.83$ & 0.660 & 0.001 & $79 \%$ \\
\hline & Caucasians & 3 & 0.88 & $0.41,1.89$ & 0.740 & 0.070 & $62 \%$ \\
\hline & Overall & 8 & 1.01 & $0.83,1.23$ & 0.930 & 0.000 & $80 \%$ \\
\hline & HWE (yes) & 7 & 0.96 & $0.77,1.21$ & 0.750 & 0.000 & $77 \%$ \\
\hline & $\mathrm{PB}$ & 3 & 1.14 & $0.90,1.45$ & 0.280 & 0.020 & $73 \%$ \\
\hline \multirow[t]{5}{*}{ C vs. A } & $\mathrm{HB}$ & 5 & 0.95 & $0.73,1.22$ & 0.680 & 0.008 & $71 \%$ \\
\hline & Large sample & 3 & 1.13 & $0.90,1.43$ & 0.290 & 0.005 & $81 \%$ \\
\hline & Small sample & 5 & 0.92 & $0.67,1.27$ & 0.620 & 0.005 & $73 \%$ \\
\hline & Asians & 5 & 1.04 & $0.79,1.37$ & 0.790 & 0.000 & $86 \%$ \\
\hline & Caucasians & 3 & 0.97 & $0.75,1.26$ & 0.820 & 0.180 & $42 \%$ \\
\hline
\end{tabular}


Table 3 Summary of odds ratios (95\% Cl) in the analysis of the relationship between VEGF gene polymorphisms in rs2010963 and coronary artery disease susceptibility

\begin{tabular}{|c|c|c|c|c|c|c|c|}
\hline \multirow[t]{2}{*}{ Genetic Model } & \multirow{2}{*}{$\begin{array}{l}\text { Overall and } \\
\text { Subgroups }\end{array}$} & \multirow[t]{2}{*}{$\mathrm{N}$} & \multicolumn{3}{|c|}{ Test of Association } & \multicolumn{2}{|c|}{ Test of Heterogeneity } \\
\hline & & & $\overline{\mathrm{OR}}$ & $95 \% \mathrm{Cl}$ & $\overline{P_{\text {-value }}}$ & $P_{\text {Heterogeneity }}$ & $1^{2}(\%)$ \\
\hline & Overall & 10 & 1.18 & $0.94,1.48$ & 0.140 & 0.002 & $65 \%$ \\
\hline & PB & 3 & 1.31 & $0.77,2.25$ & 0.320 & 0.020 & $74 \%$ \\
\hline \multirow[t]{7}{*}{$C C+G C$ vs. GG } & $\mathrm{HB}$ & 7 & 1.13 & $0.88,1.44$ & 0.340 & 0.010 & $62 \%$ \\
\hline & Large sample size & 4 & 1.11 & $0.77,1.58$ & 0.580 & 0.009 & $74 \%$ \\
\hline & Small sample size & 6 & 1.25 & $0.92,1.68$ & 0.150 & 0.030 & $60 \%$ \\
\hline & Asians & 6 & 1.22 & $0.93,1.59$ & 0.140 & 0.010 & $67 \%$ \\
\hline & Caucasians & 4 & 1.09 & $0.68,1.76$ & 0.720 & 0.010 & $72 \%$ \\
\hline & Overall & 10 & 1.45 & $1.03,2.05$ & 0.030 & 0.000 & $74 \%$ \\
\hline & PB & 3 & 1.26 & $0.64,2.48$ & 0.500 & 0.050 & $67 \%$ \\
\hline \multirow[t]{7}{*}{ CC vs. GG + GC } & $\mathrm{HB}$ & 7 & 1.56 & $0.97,2.53$ & 0.070 & 0.000 & $79 \%$ \\
\hline & Large sample size & 4 & 1.43 & $0.74,2.74$ & 0.290 & 0.000 & $72 \%$ \\
\hline & Small sample size & 6 & 1.52 & $1.01,2.33$ & 0.040 & 0.050 & $55 \%$ \\
\hline & Asians & 6 & 1.50 & $0.98,2.31$ & 0.060 & 0.000 & $82 \%$ \\
\hline & Caucasians & 4 & 1.35 & $0.70,2.60$ & 0.370 & 0.110 & $51 \%$ \\
\hline & Overall & 10 & 1.18 & $0.90,1.57$ & 0.240 & 0.000 & $76 \%$ \\
\hline & PB & 3 & 1.32 & $0.88,1.98$ & 0.180 & 0.130 & $51 \%$ \\
\hline \multirow[t]{7}{*}{ GC vs. GG } & $\mathrm{HB}$ & 7 & 1.14 & $0.79,1.64$ & 0.490 & 0.000 & $81 \%$ \\
\hline & Large sample size & 4 & 1.28 & $0.72,2.28$ & 0.400 & 0.060 & $65 \%$ \\
\hline & Small sample size & 6 & 1.14 & $0.89,1.46$ & 0.290 & 0.190 & $32 \%$ \\
\hline & Asians & 6 & 1.25 & $0.85,1.84$ & 0.260 & 0.000 & $83 \%$ \\
\hline & Caucasians & 4 & 1.08 & $0.72,1.63$ & 0.710 & 0.060 & $59 \%$ \\
\hline & Overall & 10 & 1.57 & $1.02,2.42$ & 0.040 & 0.000 & $77 \%$ \\
\hline & PB & 3 & 1.47 & $0.60,3.63$ & 0.400 & 0.020 & $74 \%$ \\
\hline \multirow[t]{7}{*}{ CC vs. GG } & $H B$ & 7 & 1.60 & $0.93,2.77$ & 0.090 & 0.000 & $81 \%$ \\
\hline & Large sample size & 4 & 1.24 & $0.68,2.28$ & 0.480 & 0.000 & $71 \%$ \\
\hline & Small sample size & 6 & 2.03 & $1.26,3.28$ & 0.007 & 0.070 & $50 \%$ \\
\hline & Asians & 6 & 1.62 & $0.94,2.79$ & 0.080 & 0.000 & $84 \%$ \\
\hline & Caucasians & 4 & 1.40 & $0.42,4.61$ & 0.580 & 0.002 & $73 \%$ \\
\hline & Overall & 10 & 1.19 & $0.98,1.44$ & 0.080 & 0.000 & $78 \%$ \\
\hline & PB & 3 & 1.15 & $0.77,1.73$ & 0.500 & 0.006 & $81 \%$ \\
\hline \multirow[t]{5}{*}{ C vs. G } & $\mathrm{HB}$ & 7 & 1.20 & $0.94,1.53$ & 0.140 & 0.000 & $80 \%$ \\
\hline & Large sample size & 4 & 1.10 & $0.84,1.43$ & 0.490 & 0.001 & $814 \%$ \\
\hline & Small sample size & 6 & 1.27 & $1.03,1.78$ & 0.030 & 0.010 & $72 \%$ \\
\hline & Asians & 6 & 1.24 & $0.98,1.58$ & 0.070 & 0.000 & $83 \%$ \\
\hline & Caucasians & 4 & 1.08 & $0.73,1.60$ & 0.690 & 0.006 & $76 \%$ \\
\hline
\end{tabular}

Abbreviations: $N$ number of studies; $O R$ odds ratio; $C l$ confidence interval; $H B$ hospital-based; $P B$ population-based; $P_{-V a l u e r} P$ value for association; $P$ Heterogeneity, $P$ value for heterogeneity

= 1.38, 95\% CI: 0.98-1.93; CT vs. CC: OR =1.24, 95\% CI: 0.86-1.80; T vs. C: $\mathrm{OR}=1.23,95 \% \mathrm{CI}$ : 0.93-1.62) (Table 4; Additional file 1: Figs. S11-S15). The subgroup analysis stratified by ethnicity indicated that the rs3025039 polymorphism was significantly associated with CAD in Asian populations compared to Caucasian populations for all genetic models, except for the dominant and recessive genetic models. Stratification by sample size indicated that the rs3025039 polymorphism was significantly associated with CAD for large sample sizes in the homozygous genetic model. No significant associations were observed in the subgroup analysis stratified by the control source (Table 4). 
Table 4 Summary of odds ratios (95\% Cl) in the analysis of the relationship between VEGF gene polymorphisms in rs3025039 and coronary artery disease susceptibility

\begin{tabular}{|c|c|c|c|c|c|c|c|}
\hline \multirow[t]{2}{*}{ Genetic Model } & \multirow{2}{*}{$\begin{array}{l}\text { Overall and } \\
\text { Subgroups }\end{array}$} & \multirow[t]{2}{*}{$\mathrm{N}$} & \multicolumn{3}{|c|}{ Test of Association } & \multicolumn{2}{|c|}{ Test of Heterogeneity } \\
\hline & & & $\overline{\mathrm{OR}}$ & $95 \% \mathrm{Cl}$ & $P_{\text {-value }}$ & $P_{\text {Heterogeneity }}$ & $1^{2}(\%)$ \\
\hline & Overall & 8 & 1.26 & $0.89,1.79$ & 0.200 & 0.000 & $84 \%$ \\
\hline & HWE (yes) & 7 & 1.14 & $0.86,1.50$ & 0.360 & 0.020 & $59 \%$ \\
\hline & PB & 2 & 1.24 & $0.32,4.77$ & 0.750 & 0.000 & $91 \%$ \\
\hline \multirow[t]{8}{*}{$T T+C T$ vs. CC } & $\mathrm{HB}$ & 6 & 1.17 & $0.82,1.65$ & 0.390 & 0.020 & $63 \%$ \\
\hline & Large sample size & 2 & 1.55 & $0.67,3.54$ & 0.300 & 0.000 & $96 \%$ \\
\hline & Small sample size & 6 & 1.15 & $0.76,1.75$ & 0.500 & 0.008 & $71 \%$ \\
\hline & Asians & 4 & 1.17 & $0.82,1.65$ & 0.390 & 0.020 & $63 \%$ \\
\hline & Caucasians & 4 & 0.91 & $0.69,1.20$ & 0.40 & 0.490 & $0 \%$ \\
\hline & Overall & 8 & 1.38 & $0.98,1.93$ & 0.060 & 0.950 & $0 \%$ \\
\hline & HWE (yes) & 7 & 1.35 & $0.88,2.05$ & 0.170 & 0.910 & $0 \%$ \\
\hline & PB & 2 & 1.34 & $0.78,2.29$ & 0.290 & 0.430 & $0 \%$ \\
\hline \multirow[t]{8}{*}{ TT vs. $C C+C T$} & $\mathrm{HB}$ & 6 & 1.40 & $0.91,2.17$ & 0.130 & 0.910 & $0 \%$ \\
\hline & Large sample size & 2 & 1.37 & $0.88,2.14$ & 0.170 & 0.790 & $0 \%$ \\
\hline & Small sample size & 6 & 1.40 & $0.91,2.17$ & 0.130 & 0.910 & $0 \%$ \\
\hline & Asians & 4 & 1.45 & $0.99,2.12$ & 0.060 & 0.920 & $0 \%$ \\
\hline & Caucasians & 4 & 1.14 & $0.55,2.36$ & 0.730 & 0.710 & $0 \%$ \\
\hline & Overall & 8 & 1.24 & $0.86,1.80$ & 0.250 & 0.000 & $84 \%$ \\
\hline & HWE (yes) & 7 & 1.11 & $0.84,1.47$ & 0.440 & 0.030 & $58 \%$ \\
\hline & PB & 2 & 1.27 & $0.33,4.95$ & 0.730 & 0.001 & $91 \%$ \\
\hline \multirow[t]{8}{*}{ CT vs. CC } & $H B$ & 6 & 1.18 & $0.89,1.56$ & 0.250 & 0.040 & $58 \%$ \\
\hline & Large sample size & 2 & 1.55 & $0.64,3.72$ & 0.330 & 0.000 & $96 \%$ \\
\hline & Small sample size & 6 & 1.18 & $0.89,1.56$ & 0.250 & 0.040 & $58 \%$ \\
\hline & Asians & 4 & 1.64 & $1.01,2.69$ & 0.050 & 0.000 & $89 \%$ \\
\hline & Caucasians & 4 & 0.89 & $0.66,1.19$ & 0.410 & 0.640 & $0 \%$ \\
\hline & Overall & 8 & 1.55 & $1.10,2.17$ & 0.010 & 0.830 & $0 \%$ \\
\hline & HWE (yes) & 7 & 1.39 & $0.91,2.13$ & 0.120 & 0.820 & $0 \%$ \\
\hline & PB & 2 & 1.67 & $0.98,2.85$ & 0.060 & 0.260 & $21 \%$ \\
\hline \multirow[t]{8}{*}{ TT vs. CC } & $\mathrm{HB}$ & 6 & 1.47 & $0.95,2.28$ & 0.080 & 0.830 & $0 \%$ \\
\hline & Large sample size & 2 & 1.61 & $1.03,2.53$ & 0.040 & 0.410 & $0 \%$ \\
\hline & Small sample size & 6 & 1.47 & $0.95,2.28$ & 0.080 & 0.830 & $0 \%$ \\
\hline & Asians & 4 & 1.70 & $1.16,2.50$ & 0.007 & 0.810 & $0 \%$ \\
\hline & Caucasians & 4 & 1.10 & $0.53,2.28$ & 0.810 & 0.650 & $0 \%$ \\
\hline & Overall & 8 & 1.23 & $0.93,1.62$ & 0.140 & 0.000 & $80 \%$ \\
\hline & HWE (yes) & 7 & 1.13 & $0.91,1.42$ & 0.270 & 0.040 & $54 \%$ \\
\hline & PB & 2 & 1.17 & $0.38,3.57$ & 0.780 & 0.002 & $90 \%$ \\
\hline \multirow[t]{5}{*}{ T vs. C } & $\mathrm{HB}$ & 6 & 1.19 & $0.96,1.48$ & 0.110 & 0.070 & $51 \%$ \\
\hline & Large sample size & 2 & 1.43 & $0.76,2.70$ & 0.270 & 0.000 & $95 \%$ \\
\hline & Small sample size & 6 & 1.19 & $0.96,1.48$ & 0.110 & 0.070 & $51 \%$ \\
\hline & Asians & 4 & 1.50 & $1.06,2.13$ & 0.020 & 0.000 & $85 \%$ \\
\hline & Caucasians & 4 & 0.94 & $0.74,1.21$ & 0.640 & 0.390 & $0 \%$ \\
\hline
\end{tabular}


Table 5 Summary of odds ratios $(95 \% \mathrm{Cl})$ in the analysis of the relationship between VEGF gene polymorphisms in rs1570360 and coronary artery disease susceptibility

\begin{tabular}{|c|c|c|c|c|c|c|}
\hline \multirow[t]{2}{*}{ Genetic Model } & \multirow[t]{2}{*}{ N } & \multicolumn{3}{|c|}{ Test of Association } & \multicolumn{2}{|c|}{ Test of Heterogeneity } \\
\hline & & $\overline{\mathrm{OR}}$ & $95 \% \mathrm{Cl}$ & $P_{\text {-value }}$ & $P_{\text {Heterogeneity }}$ & $I^{2}(\%)$ \\
\hline$A A+G A$ vs. GG & 3 & 1.52 & $0.77,3.00$ & 0.220 & 0.000 & $89 \%$ \\
\hline$A A$ vs. $G G+G A$ & 3 & 1.40 & $0.62,3.15$ & 0.410 & 0.004 & $82 \%$ \\
\hline GA vs. GG & 3 & 1.41 & $0.91,2.18$ & 0.120 & 0.040 & $70 \%$ \\
\hline AA vs. GG & 3 & 1.70 & $0.50,5.73$ & 0.390 & 0.000 & $91 \%$ \\
\hline A vs. G & 3 & 1.32 & $0.81,2.16$ & 0.260 & 0.000 & $90 \%$ \\
\hline
\end{tabular}

Abbreviations: $N$ number of studies; $O R$ odds ratio; $C l$ confidence interval; $P_{-}$ ${ }_{\text {value, }} P$ value for association; $P$ Heterogeneity, $P$ value for heterogeneity

\section{Association between the rs 1570360 polymorphism and susceptibility to CAD}

No significant associations were observed between the rs1570360 polymorphism and CAD susceptibility in all genetic models (Table 5). Due to the limited number of eligible studies on rs1570360, no further subgroup analysis was performed.

\section{Sensitivity analysis}

The influence of each study on the overall meta-analysis was evaluated by excluding each study at a time. The sensitivity analysis confirmed the stability of our meta-analysis and indicated that no individual study significantly affected the pooled result (Fig. 2).

\section{Publication Bias}

We performed funnel plots and Egger's test to assess the publication bias of the included studies. A funnel plot was not constructed for the 3 rs1570360 studies. The funnel plot distribution of distinct studies appeared nearly symmetrical (Fig. 3). Moreover, Egger's test failed to show statistically significant asymmetry in dominant genetic models (rs699947: $t=-1.21, P=0.270$; rs2010963: $t=-$ 0.62, $P=0.551$; rs3025039: $t=-2.07, P=0.086$; rs1570360: $t=-3.05, P=0.787$ ).

\section{Discussion}

VEGFs have been reported to alleviate complications closely linked to CAD by promoting the recanalization of thrombus-blocked blood vessels, establishing collateral circulation against myocardial ischaemia, and improving endothelium-dependent vasodilatation [9, 34, 35]. However, despite the important impacts of hereditary factors on CAD development, a link between VEGF SNPs and CAD risk has yet to be sufficiently elucidated.

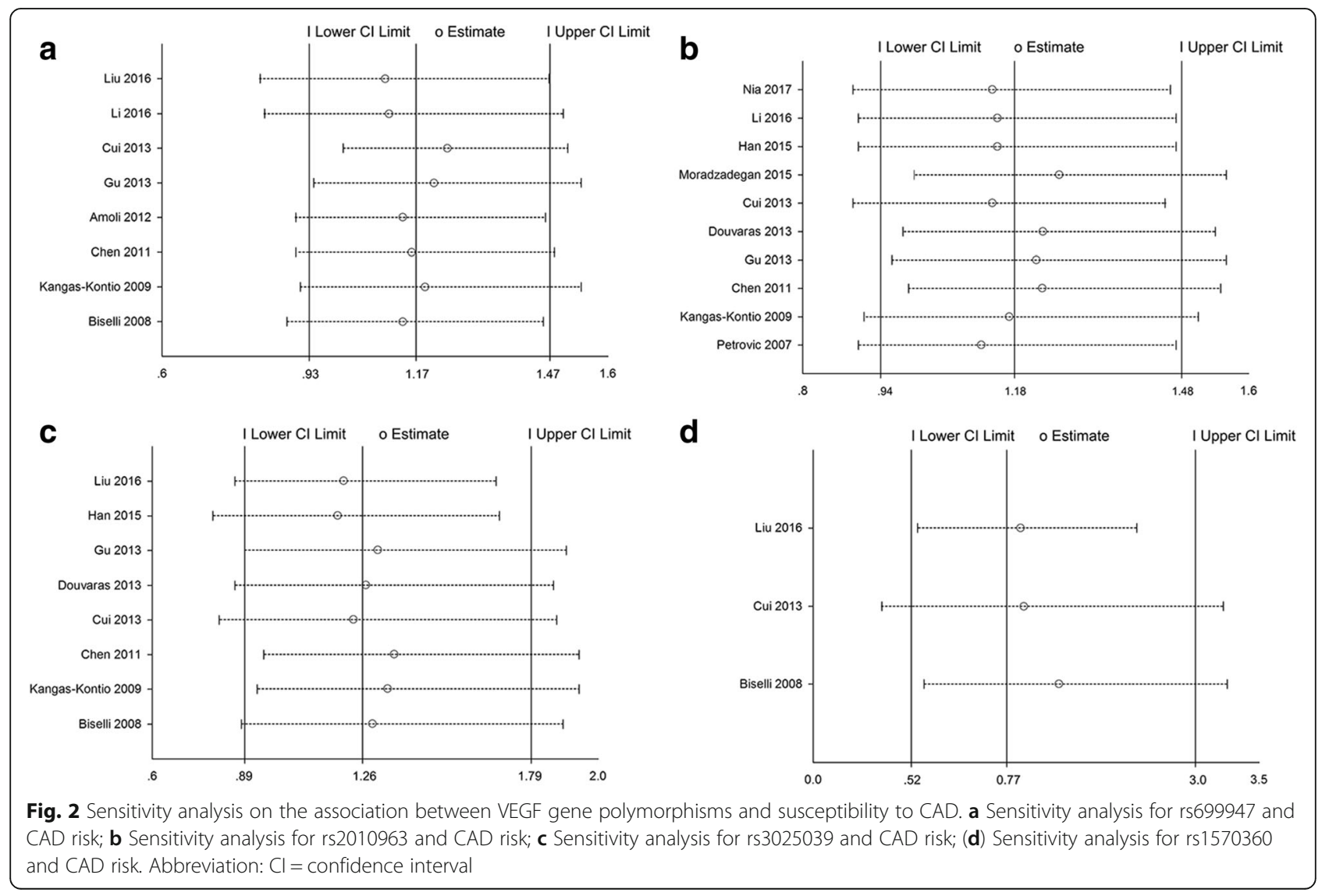




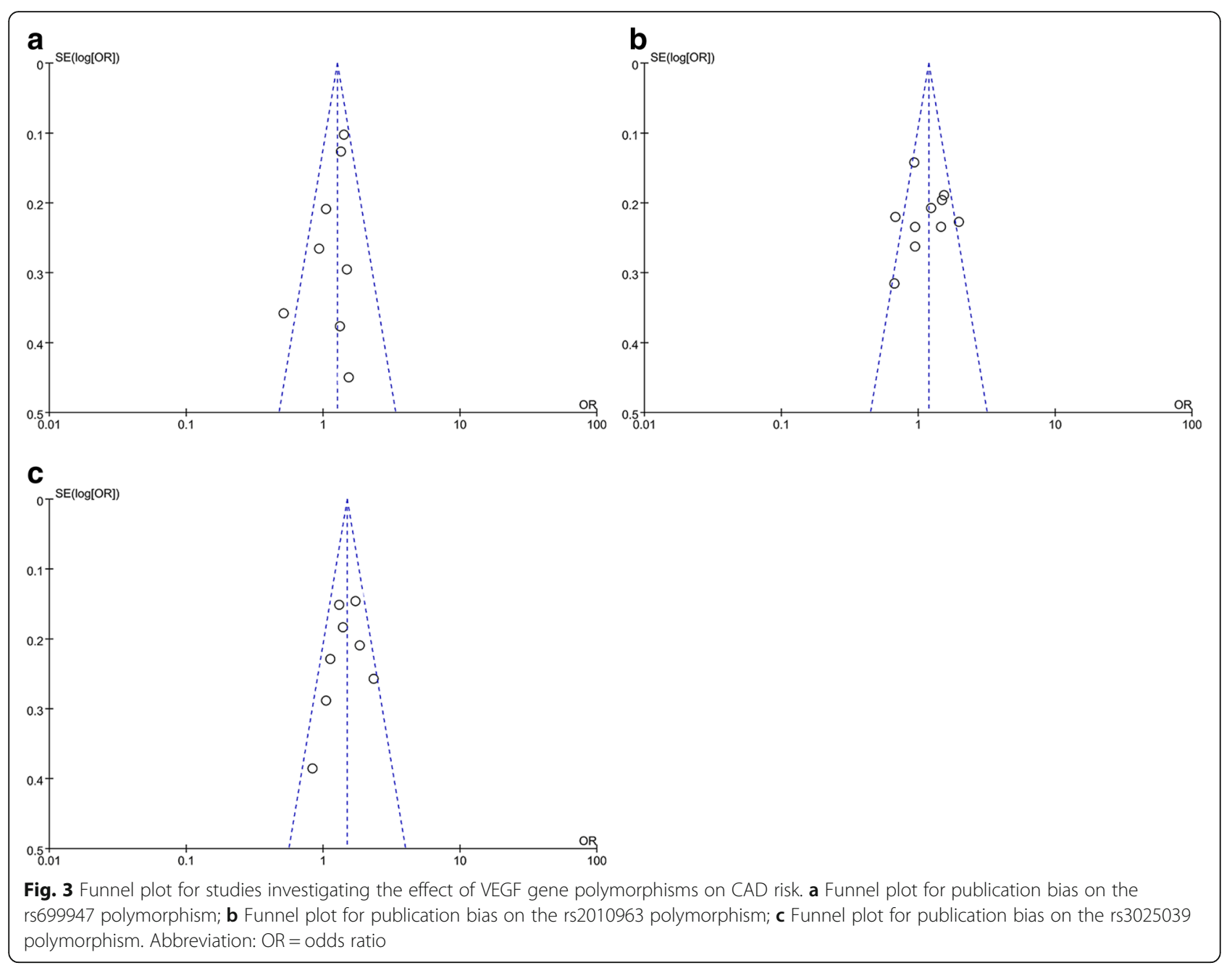

In this meta-analysis, we analysed 29 eligible studies and found that rs699947, rs2010963, and rs3025020 polymorphisms increased CAD susceptibility, suggesting that these polymorphisms may be risk factors for CAD. However, the rs1570360 polymorphism failed to yield an association with $\mathrm{CAD}$. One possible explanation for this finding is that the functional polymorphisms of rs699947, rs2010963, and rs3025020 may have more profound effects on angiogenesis than other SNPs. This discrepancy may also result in inter-individual differences in CAD incidence. The findings reported here are in agreement with some studies [21, 28] but not others [23]. The estimated pooled OR did not obviously change when non-HWE studies were excluded, suggesting the stability of the results. Subgroup analysis based on ethnicity identified an association between VEGF gene (rs699947 and rs3025039) polymorphisms and CAD, especially in Asian populations. This discrepancy may be due to genetic heterogeneity among different ethnicities. Moreover, to relieve heterogeneity bias within Asian and small sample size subgroups, stratified analysis was performed and suggested that ethnicity and sample size may be potential sources of heterogeneity. In addition, NOS quality assessment showed no obvious publication bias in our study, which supports the reliability of the conclusions.

Whether VEGF is a pro-atherosclerotic or anti-atherosclerotic factor is currently under debate. Some studies have reported that VEGF plays a role in blood vessel growth and the regulation of vessel wall integrity by promoting regeneration of endothelial cells, enhancing endothelial function, and slowing smooth muscle differentiation [34, 36, 37]. Grosskreutz et al. [38] observed that VEGF expression accelerates re-endothelialisation and reduces intimal thickening and thrombus formation. Howell et al. [39] also reported that higher VEGF expression has a protective effect in atherosclerosis development. In contrast, other studies have shown that VEGF induces atherosclerosis via promotion of vascular smooth muscle cell proliferation and plaque neovascularization [35, 40]. Eaton et al. [41] reported that elevated levels of VEGF were significantly associated with CAD mortality after 13 years of follow-up. Additionally, recent evidence has suggested that low VEGF levels may 
be needed to maintain vascular homeostasis, whereas upregulated VEGF has been observed in active angiogenesis processes during acute or stable ischaemic myocardium [42-44]. Overall, these data suggest that changes in VEGF levels may reflect the progressive stages of angiogenesis activity.

Several association studies have reported that the + $405 \mathrm{C} / \mathrm{G},+936 \mathrm{C} / \mathrm{T}$, and $-2578 \mathrm{~A} / \mathrm{C}$ polymorphisms have an impact on VEGF protein synthesis [12, 39, 45]. However, several functional SNPs in the VEGF gene may contribute to CAD development, possibly by altering VEGF expression or protein activity. It is important to note that several stimulatory factors associated with hypoxia, oxidative stress, hyperglycaemia, hormones, and cytokines can influence plasma VEGF levels [9, 24, 46]. Furthermore, since $2578 \mathrm{~A} / \mathrm{C}$ is in strong linkage disequilibrium with 1154G/A, carriers of the risk allele $-2578 \mathrm{C}$ will in most cases also be carriers of the - 1154 An allele, which appears to lead to enhanced VEGF expression [47]. Based on the above analysis, we speculate that variations in the VEGF gene may be susceptibility factors and outcome predictors for CAD.

Several limitations in our meta-analysis should be addressed. First, heterogeneity may have influenced the interpretation of our results. The limited number of studies with small sample sizes may have influenced the reliability of the conclusions; however, the heterogeneity was reduced by the subgroup analysis. Moreover, further relevant studies may supplement the present conclusions. Second, because of the limitation of the available data that were extracted from each selected study, our results were based on unadjusted estimates, which may produce misleading results. Third, despite searching comprehensive databases, only Asian and Caucasian populations were included in our meta-analysis. Ethnicity bias may exist in our analysis, and the conclusions may not be applicable to other races. Fourth, in our meta-analysis, the case group of several eligible studies consisted of patients with MI only. Most MIs occur due to CAD, despite the pathophysiology of MI is partly different from that of CAD. This discrepancy may increase the clinical heterogeneity among the studies.

\section{Conclusions}

In conclusion, the current meta-analysis supports the existence of an association between VEGF gene polymorphisms (rs699947, rs2010963, and rs3025039) and susceptibility to CAD, especially in Asian populations. Although a subgroup analysis was used to investigate the source of the heterogeneity, the results should be interpreted with caution.

\section{Additional file}

\begin{abstract}
Additional file 1: Table S1. MOOSE checklist for meta-analysis. Table S2. Methodological quality of the included studies according to the NewcastleOttawa Scale. Figure S1. Forest plot for the association between the VEGF rs699947 polymorphism and CAD risk in dominant genetic models. Figure S2. Forest plot for the association between the VEGF rs699947 polymorphism and CAD risk in recessive genetic models. Figure S3. Forest plot for the association between the VEGF rs699947 polymorphism and CAD risk in heterozygous genetic models. Figure S4. Forest plot for the association between the VEGF rs699947 polymorphism and CAD risk in homozygous genetic models. Figure S5. Forest plot for the association between the VEGF rs699947 polymorphism and CAD risk in allele genetic models. Figure S6. Forest plot for the association between the VEGF rs2010963 polymorphism and CAD risk in dominant genetic models. Figure S7. Forest plot for the association between the VEGF rs2010963 polymorphism and CAD risk in recessive genetic models. Figure S8. Forest plot for the association between the VEGF rs2010963 polymorphism and CAD risk in heterozygous genetic models. Figure S9. Forest plot for the association between the VEGF rs2010963 polymorphism and CAD risk in homozygous genetic models. Figure S10. Forest plot for the association between the VEGF rs2010963 polymorphism and $C A D$ risk in allele genetic models. Figure S11. Forest plot for the association between the VEGF rs3025039 polymorphism and CAD risk in dominant genetic models. Figure S12. Forest plot for the association between the VEGF rs3025039 polymorphism and CAD risk in recessive genetic models. Figure S13. Forest plot for the association between the VEGF rs3025039 polymorphism and CAD risk in heterozygous genetic models. Figure S14. Forest plot for the association between the VEGF rs3025039 polymorphism and CAD risk in homozygous genetic models. Figure S15. Forest plot for the association between the VEGF rs3025039 polymorphism and CAD risk in allele genetic models. (DOC $3267 \mathrm{~kb}$ )
\end{abstract}

\section{Abbreviations}

CAD: Coronary artery disease; Cls: Confidence intervals; HWE: HardyWeinberg equilibrium; MI: Myocardial infarction; MOOSE: Meta-analysis of Observational Studies in Epidemiology; NOS: Newcastle-Ottawa Scale; ORs: Odds ratios; SNPs: Single nucleotide polymorphisms; VEGF: Vascular endothelial growth factor

\section{Funding}

This work was supported by National Nature Science Foundation of China (No. 81770451). The funding body had no role in the design, collection, analysis, or interpretation of this study.

\section{Availability of data and materials}

The articles included in this meta-analysis can be found in the respective journals.

\section{Authors' contributions}

WQM was in charge of conceived and designed the study. WQM, YW and $\mathrm{XQH}$ were responsible for collection of data and performing the statistical analysis and manuscript preparation. NFL and YZ were responsible for checking the data. All authors read and approved the final version.

Ethics approval and consent to participate

Not applicable.

Consent for publication

Not applicable.

Competing interests

The authors declare that they have no competing interests.

\section{Publisher's Note}

Springer Nature remains neutral with regard to jurisdictional claims in published maps and institutional affiliations. 


\section{Received: 7 December 2017 Accepted: 18 June 2018} Published online: 04 July 2018

\section{References}

1. Mozaffarian D, Benjamin EJ, Go AS, Arnett DK, Blaha MJ, Cushman M, Das SR, de Ferranti S, Despres JP, Fullerton HJ, et al. Heart disease and stroke statistics-2016 update: a report from the American Heart Association. Circulation. 2016;133(4):e38-e360.

2. Hansson GK. Inflammation, atherosclerosis and coronary artery disease. N Engl J Med. 2005;352(16):1685-95.

3. Sitia S, Tomasoni L, Atzeni F, Ambrosio G, Cordiano C, Catapano A, Tramontana S, Perticone F, Naccarato P, Camici P, et al. From endothelial dysfunction to atherosclerosis. Autoimmun Rev. 2010;9(12):830-4.

4. Chung NA, Lydakis C, Belgore F, Li-Saw-Hee FL, Blann AD, Lip GYH. Angiogenesis, thrombogenesis, endothelial dysfunction and angiographic severity of coronary artery disease. Heart. 2003;89(12):1411-5.

5. Sena CM, Pereira AM, Seiça R. Endothelial dysfunction-a major mediator of diabetic vascular disease. Biochim Biophys Acta. 2013;1832(12):2216-31.

6. Kolluru GK, Bir SC, Kevil CG. Endothelial dysfunction and diabetes: effects on angiogenesis, vascular remodeling, and wound healing. Int J Vasc Med. 2012;2012:918267

7. Wang S, Li X, Parra M, Verdin E, Bassel-Duby R, Olson EN. Control of endothelial cell proliferation and migration by VEGF signaling to histone deacetylase 7. Proc Natl Acad Sci U S A. 2008;105(22):7738-43.

8. Inoue $M$, Itoh H, Ueda M, Naruko T, Kojima A, Komatsu R. K, Ogawa Y, Tamura N, Takaya K, et al. vascular endothelial growth factor (VEGF) expression in human coronary atherosclerotic lesions. Circulation. 1998; 98(20):2108-16

9. Ferrara N. Molecular and biological properties of vascular endothelial growth factor. J Mol Med (Berl). 1999;77(7):527-43.

10. Tamura K, Amano T, Satoh T, Saito D, Yonei-Tamura S, Yajima H. Expression of rigf, a member of avian VEGF family, correlates with vascular patterning in the developing chick limb bud. Mech Dev. 2003;120(2):199-209.

11. Brogan IJ, Khan N, Isaac K, Hutchinson JA, Pravica V, Hutchinson IV. Novel polymorphisms in the promoter and 5'UTR regions of the human vascular endothelial growth factor gene. Hum Immunol 1999; 60(12):1245-1249.

12. Ruggiero D, Dalmasso C, Nutile T, Sorice R, Dionisi L, Aversano M, Broet $P$, Leutenegger AL, Bourgain C, Ciullo M. Genetics of VEGF serum variation in human isolated populations of cilento: importance of VEGF polymorphisms. PLoS One. 2011;6(2):e16982.

13. Pantsulaia I, Trofimov S, Kobyliansky E, Livshits G. Heritability of circulating growth factors involved in the angiogenesis in healthy human population. Cytokine. 2004:27(6):152-8.

14. Al-Habboubi HH, Sater MS, Almawi AW, Al-Khateeb GM, Almawi WY. Contribution of VEGF polymorphisms to variation in VEGF serum levels in a healthy population. Eur Cytokine Netw. 2011;22(3):154-8.

15. Hofstaetter JG, Saad FA, Sunk IG, Bobacz K, Friehs I, Glimcher MJ. Agedependent expression of VEGF isoforms and receptors in the rabbit anterior cruciate ligament. Biochim Biophys Acta. 2007;1770(7):997-1002.

16. Hu GL, Ma G, Ming JH. Impact of common SNPs in VEGF gene on the susceptibility of osteosarcoma. Genet Mol Res. 2015;14(4):14561-6.

17. Jiang $Y$, Liang $G$, Wang $L$, Jiang J, Du G, Huang $Y$. Association between vascular endothelial growth factor $+936 \mathrm{C} / \mathrm{T}$ gene polymorphism and age-related macular degeneration. J Int Med Res. 2013:41(2):317-24.

18. Kim HW, Ko GJ, Kang YS, Lee MH, Song HK, Kim HK, Cha DR. Role of the VEGF $936 \mathrm{C} / \mathrm{T}$ polymorphism in diabetic microvascular complications in type 2 diabetic patients. Nephrology (Carlton). 2009;14(7):681-8.

19. Wei H, Liu L, Chen Q. Selective removal of mitochondria via mitophagy: distinct pathways for different mitochondrial stresses. Biochim Biophys Acta. 2015;1853(10 Pt B):2784-90.

20. Xian W, Zheng H, Wu WJ. Predictive value of vascular endothelial growth factor polymorphisms on the risk of renal cell carcinomas. Genet Mol Res. 2015;14(3):7634-42.

21. Han X, Liu L, Niu J, Yang J, Zhang Z, Zhang Z. Association between VEGF polymorphisms (936C/T, -460T/C and -634G/C) with haplotypes and coronary heart disease susceptibility. Int J Clin Exp Pathol. 2015;8(1):922-7.

22. Li L, Pan Y, Dai L, Liu B, Zhang D. Association of genetic polymorphisms on vascular endothelial growth factor and its receptor genes with susceptibility to coronary heart disease. Med Sci Monit. 2016;22:31-40.
23. Biselli PM, Guerzoni AR, de Godoy MF, Pavarino-Bertelli EC, Goloni-Bertollo EM. Vascular endothelial growth factor genetic variability and coronary artery disease in Brazilian population. Heart Vessel. 2008;23(6):371-5.

24. Petrovic D, Verhovec R, Globocnik Petrovic M, Osredkar J, Peterlin B. Association of vascular endothelial growth factor gene polymorphism with myocardial infarction in patients with type 2 diabetes. Cardiology. 2007; 107(4):291-5.

25. Kangas-Kontio T, Tapanainen JM, Huikuri H, Savolainen ER, Paivansalo M, Kauma H, Kesaniemi YA, Savolainen MJ, Kakko S. Variation in the vascular endothelial growth factor gene, carotid intima-media thickness and the risk of acute myocardial infarction. Scand J Clin Lab Invest. 2009;69(3):335-43.

26. Chen Y, Dawes PT, Packham JC, Mattey DL. Interaction between smoking and polymorphism in the promoter region of the VEGFA gene is associated with ischemic heart disease and myocardial infarction in rheumatoid arthritis. J Rheumatol. 2011;38(5):802-9.

27. Amoli MM, Amiri P, Alborzi A, Larijani B, Saba S, Tavakkoly-Bazzaz J. VEGF gene mRNA expression in patients with coronary artery disease. Mol Biol Rep. 2012;39(9):8595-9.

28. Cui QT, Li Y, Duan CH, Zhang W, Guo XL. Further evidence for the contribution of the vascular endothelial growth factor gene in coronary artery disease susceptibility. Gene. 2013:521(2):217-21.

29. Gu H, Chen W, Yin J, Chen S, Zhang J, Gong J. Methionine sulfoxide reductase a rs10903323 G/a polymorphism is associated with increased risk of coronary artery disease in a Chinese population. Clin Biochem. 2013; 46(16-17):1668-72.

30. Moradzadegan A, Vaisi-Raygani A, Nikzamir A, Rahimi Z. Angiotensin converting enzyme insertion/deletion (I/D) (rs4646994) and VEGF polymorphism (+405G/C; rs2010963) in type II diabetic patients: association with the risk of coronary artery disease. J Renin-Angiotensin-Aldosterone Syst. 2015;16(3):672-80.

31. Liu D, Song J, Ji X, Liu Z, Cong M, Hu B. Association of genetic polymorphisms on VEGFA and VEGFR2 with risk of coronary heart disease. Medicine (Baltimore). 2016;95(19):e3413.

32. Douvaras P, Antonatos DG, Kekou K, Patsilinakos S, Chouliaras G, Christou A Andrikou A, Kanavakis E. Association of VEGF gene polymorphisms with the development of heart failure in patients after myocardial infarction. Cardiology. 2009;114(1):11-8.

33. Kalayi Nia S, Ziaee S, Boroumand MA, Sotudeh Anvari M, Pourgholi L, Jalali A. The impact of vascular endothelial growth factor $+405 \mathrm{C} / \mathrm{G}$ polymorphism on long-term outcome and severity of coronary artery disease. J Clin Lab Anal. 2017;31(4)

34. Coultas L, Chawengsaksophak K, Rossant J. Endothelial cells and VEGF in vascular development. Nature. 2005:438(7070):937-45.

35. Grunewald M, Avraham I, Dor Y, Bachar-Lustig E, Itin A, Jung S, Chimenti S, Landsman L, Abramovitch R, Keshet E. VEGF-induced adult neovascularization: recruitment, retention, and role of accessory cells. Cell. 2006;124(1):175-89.

36. Azambuja AP, Portillo-Sanchez V, Rodrigues MV, Omae SV, Schechtman D, Strauss BE, Costanzi-Strauss E, Krieger JE, Perez-Pomares JM, Xavier-Neto J. Retinoic acid and VEGF delay smooth muscle relative to endothelial differentiation to coordinate inner and outer coronary vessel wall morphogenesis. Circ Res. 2010;107(2):204-16.

37. Kliche $S$. Waltenberger J. VEGF receptor signaling and endothelial function. IUBMB Life. 2001:52(1-2):61-6.

38. Grosskreutz CL, Anand-Apte B, Duplaa C, Quinn TP, Terman Bl, Zetter B, D'Amore PA. Vascular endothelial growth factor-induced migration of vascular smooth muscle cells in vitro. Microvasc Res. 1999;58(2):128-36.

39. Howell WM, Ali S, Rose-Zerilli MJ, Ye S. VEGF polymorphisms and severity of atherosclerosis. J Med Genet. 2005;42(6):485-90.

40. Osada-Oka M, Ikeda T, Imaoka S, Akiba S, Sato T. VEGF-enhanced proliferation under hypoxia by an autocrine mechanism in human vascular smooth muscle cells. J Atheroscler Thromb. 2008;15(1):26-33.

41. Eaton CB, Gramling R, Parker DR, Roberts MB, Lu B, Ridker PM. Prospective association of vascular endothelial growth factor-a (VEGF-A) with coronary heart disease mortality in southeastern New England. Atherosclerosis. 2008; 200(1):221-7.

42. Fuchs S, Lavi I, Tzang O, Bessler H, Brosh D, Bental T, Dvir D, Einav S, Kornowski R. Intracoronary monocyte chemoattractant protein 1 and vascular endothelial growth factor levels are associated with necrotic core, calcium and fibrous tissue atherosclerotic plaque components: an intracoronary ultrasound radiofrequency study. Cardiology. 2012;123(2): 125-32. 
43. Lee KW, Lip GY, Blann AD. Plasma angiopoietin-1, angiopoietin-2, angiopoietin receptor tie-2, and vascular endothelial growth factor levels in acute coronary syndromes. Circulation. 2004;110(16):2355-60.

44. Zygalaki E, Kaklamanis L, Nikolaou NI, Kyrzopoulos S, Houri M, Kyriakides Z, Lianidou ES, Kremastinos DT. Expression profile of total VEGF, VEGF splice variants and VEGF receptors in the myocardium and arterial vasculature of diabetic and non-diabetic patients with coronary artery disease. Clin Biochem. 2008;41(1-2):82-7.

45. Watson CJ, Webb NJ, Bottomley MJ, Brenchley PE. Identification of polymorphisms within the vascular endothelial growth factor (VEGF) gene: correlation with variation in VEGF protein production. Cytokine. 2000;12(8):1232-5.

46. Kim B-S, Chen J, Weinstein T, Noiri E, Goligorsky MS. VEGF expression in hypoxia and hyperglycemia: reciprocal effect on branching angiogenesis in epithelial-endothelial co-cultures. J Am Soc Nephrol. 2002;13(8):2027-36.

47. Mohammadi M, Bazrafshani MR, Day PJ, Ollier WE. Vascular endothelia growth factor production is regulated by gene polymorphisms. Iran J Immunol. 2009;6(3):119-29.

Ready to submit your research? Choose BMC and benefit from:

- fast, convenient online submission

- thorough peer review by experienced researchers in your field

- rapid publication on acceptance

- support for research data, including large and complex data types

- gold Open Access which fosters wider collaboration and increased citations

- maximum visibility for your research: over $100 \mathrm{M}$ website views per year

At BMC, research is always in progress.

Learn more biomedcentral.com/submissions 